\title{
Median Frequency Analysis of Shoulder Muscles Using EMG Power Spectrum Analysis After Rotator Cuff Repair
}

\author{
Jeong-Il Kang \\ Department of Physical Therapy \\ Sehan University, South Korea \\ Young-Jun Moon \\ Department of Physical Therapy, Graduate School \\ Sehan University, South Korea
}

\begin{abstract}
The purpose of this study was to analyze the changes in muscle fatigue by using electromyography (EMG) measurements of the median frequency of shoulder muscles of patients who underwent rotator cuff repair, and to provide basic data for efficient exercise methods during rehabilitation after the repair. In the study, 24 male and 8 female patients who underwent rotator cuff repair performed continuous passive shoulder joint motion for 3 weeks and the median frequency of their shoulder muscles before and after the repair were compared using measurements by EMG. The infraspinatus, supraspinatus, and serratus anterior muscles showed significant differences in the changes in the median frequency before and after the repair. Significant differences were also found in the median frequency of the three muscles both before and after the repair. Additionally, the middle deltoid muscle had a lower median frequency than the supraspinatus muscle before the repair and the infraspinatus muscle had a lower median frequency than the middle deltoid muscle after the repair. It is expected that these results will serve as important data in developing rehabilitation exercise programs for the infraspinatus, supraspinatus, and serratus anterior muscles by compensating the middle deltoid muscle with the lowest muscle fatigue after the rotator cuff repair erase.
\end{abstract}

Key words: Continuous Passive Movement, Electromyography, Median Frequency, Muscle Fatique, Rotator Cuff.

\section{INTRODUCTION}

Rotator Cuff Tear is a result of normal aging process and occurred more frequently in aged population and people getting involved in sports activitied [1], and one of the most common diseases that cause pain in shoulder blade and dysfunction in daily activities [2]. This condition is not life-threatening by rupture, but causes considerable pain, weakens muscle, and restrains movement [3].

Rotator Cuff Tear is caused by multiple factors or complex causes [1] and does not show any symptoms and breaks out without pain, and there are one or more discontinuities in muscle or tendon, and may be a result of more-than-one-year degeneration and external injury. This may appear as a result of progression of injury like impingement syndrome [4]. If rotator cuff tear occurs, the upper side of the shoulder is crepitant during passive motion of shoulder, and the passive motion is mostly normal, but active motion is limited,

\footnotetext{
* Corresponding author, Email: tkfkdgo0328@naver.com Manuscript received Jun. 16, 2014; revised Sep. 16, 2014; accepted Sep. 23, 2014
}

and it causes sleep disorder due to severe hypnalgia, which disrupts daily activity [5].

Of rotator cuff tear muscles, supraspinous muscle and infraspinous muscle are the important muscles which are responsible for shoulder stability and motion while performing tasks or involving various activities [6], and in case of supraspinous muscle tear, there appears to be a reduction in extension of shoulder joint and in upward rotation of shoulder bone due to excessive upward glide of the head of humerus and pain occurred when raising the shoulder joint initially [7], and in case of infraspinous muscle tear, the head of humerus is abnormally located [8] and thus shows abnormal pattern in moving shoulder because of loss in the process that force is generated [9]. Such false patterns cause fatigue in the surrounding muscles and may transform the suppression of central activity in the nervous system and thus appropriate treatment is necessary [10].

The conservative treatment method of rotator cuff tear includes drug therapy like painkiller and anti-inflammatory drugs for relieving pain, changes in way of living, and physical therapy. Despite such non-surgical treatment, symptoms may last for 4 to 6 months, and in this case, surgical treatment is used [11]. If surgical treatment is performed, Arthroscopic 
Salvage Repair among the surgical trearments is preferred [12], and the advantages of such an arthroscopic surgery include inspection of glenohumeral joint without separating deltoid muscle, capability of solution of lesions in internal joints, and small portion of incision that leads to small damages to soft tissue and little surgery scar and pain after surgery which leads to quick rehabilitation and functional recovery, and in terms of patient's satisfaction, it shows an excellent result [13].

Patients who received a rotator cuff muscle repair indicated that muscular activity weakened in lower extremities even with no weight bearing because the surgical site was fixated and it brought about changes in myotility [14], and Downie and Miller [15] reported that after repair, tendon and muscular atrophy increased the fatigue in the surrounding muscles over time.

Muscle fatigue hinders daily life movement and maintenance of maximum muscular contraction, damages to inputting feedback on proprioceptive sense and vision, and makes it difficult to keep a stable posture [16]. Muscular contraction is a process that the nerve action potential occurred from the cerebrum is conveyed to the extremities through the nervous system before generating muscular action potential, which is conveyed to the skeletal muscle tissue [17], and if sources of energy cannot be provided during muscular contraction for such reasons like insufficient oxygen supply into the muscle, depletion of major metabolites including source of energy, and accumulation of metabolites [18]. Scholtes et al. [19] reported that as patients who had disease suffered from high muscular fatigue, alleviation of their muscular fatigue contributed greatly to the treatment of lesions, and as therapeutic method, remedial exercise can relieve such symptoms [20]. After repair, the most commonly used remedial exercise in physical therapy department is a continuous passive shoulder joint motion, and this exercise can treat and prevent movement of tendon surface and spread of synovia at rapid rate [21] and after surgery, continuous passive shoulder joint motion should be performed to prevent stiffness of joint, protect tendon in rotator cuff, alleviate pain, and recover functions [22].

Generally, in measuring muscle fatigue, Likert scale, muscle biopsy, biochemical marker, electromyography(EMG), and many other methods have been used [23], but electromyography(EMG) has been used as a powerful tool to analyze the muscular activity related to the motion of shoulder joint [24]. The frequency spectrum of signal is expressed as fatigue evaluation scale such as mean power frequency and median frequency(MDF) and in particular, mean power frequency(MDF) is considered to be a good scale that reflects the frequency variation in electromyogram [25], and it is observed that the higher the muscular fatigue is, the low frequency range is reinforced [26]. This can be described as a reliable method in measuring because median frequency data can be obtained at a certain cycle as it is a value that can be obtained through power spectrum analysis from EMG signal obtained in experiment and with this value, muscular fatigue can be identified objectively.

Therefore, this study aims to identify the impact of tendon and muscular atrophy caused by fixation after rotator cuff repair on fatigue of surrounding muscles over time and present a foundation for studying efficient remedial exercises.

\section{MATERIALS AND METHODS}

\subsection{Materials}

Research subjects were the patients who had received a infraspinous muscle repair due to lesions caused by infraspinatus tendon 2-degree tear and injury in right rotator cuff (dominant in right side) at $\mathrm{M}$ hospital located at Cheonnam province from July 2012 to March 2013 and who showed no neurological symptoms and musculoskeletal disorders and deformities except lesions, had no pain in right upper limbs and hand, and indicated no motor disturbance. There were a total of 32 patients ( 24 males and 8 females) who were aged $40-60$ years old. They could receive outpatient treatment on a regular basis after leaving the hospital. They could understand the purpose of this study and agreed to participate in the experiment Table 1 .

Table 1. Characteristic of Subjects

\begin{tabular}{|c|c|}
\hline \multirow{2}{*}{ Items } & Experimental group(n=32) \\
\cline { 2 - 2 } & $\mathrm{M} \pm \mathrm{SD}$ \\
\hline Age & $53.67 \pm 10.44$ \\
\hline Heigth $(\mathrm{cm})$ & $168.22 \pm 6.36$ \\
\hline Weight $(\mathrm{kg})$ & $72.17 \pm 8.57$ \\
\hline Sex $(\mathrm{M} / \mathrm{F})$ & $24(75 \%) / 8(25 \%)$ \\
\hline
\end{tabular}

Descriptive Statistics

\subsection{Methods}

Research participants were asked to straighten their upper body in a specifically designed chair the previous day before reconstruction operation. They were supported with a prop when they were bending their shoulder joint by $90^{\circ}$ on the affected side, extending their elbow joint as fully as possible, and neutralizing their lower arms. They used a handgrip and concentrated strength to their utmost for 5 seconds. At this time, the median frequency value of infraspinous muscle, supraspinous muscle, serratus anterior muscle or median deltoid muscle was measured with an surface electromyogram for a preliminary inspection. After three days of repair, continuous passive motion(CPM), which is generally performed to maintain the range of joint and enhance the recovery speed when soft tissue became stiff in patients was carried out for 30 minutes, once a day and five times a week: for three weeks in total. Post-inspection was measured similarly to the preliminary inspection.

During motion, participants straightened their upper body sitting at a continuous shoulder-joint motion apparatus and extended their shoulder joint $0^{\circ}$, extended their elbow joint as fully as possible, raised their forearms when turning upside down so that shoulder joint could be bent $30^{\circ}-40^{\circ}$, and increased the extent of bending by $5^{\circ}-10^{\circ}$ on a daily basis without causing pain. The continuous shoulder-joint motion was performed at the slowest rate under strict supervision of 
physical therapist: once a day, five days a week, 30 minutes per session, for three weeks.

As data processing method for this study, Window SPSS 17.0 was used. Descriptive statistics was used for comparing the homogeneity of general characteristics in research subjects, and paired t-test was used to identify the changes in median frequency within the group. For comparing the changes in median frequency in muscles, One-way ANOVA was used. As post hoc, Tukey was used and the significance level was set at $\alpha=.05$.

\section{RESULT}

Comparison of changes in median frequency value before and after Experimental group

In comparing the changes in median frequency value in muscles before and after repair, infraspinous muscle, supraspinous muscle, serratus anterior muscle, and middle deltoid muscle were analyzed before and after that experiment. As a result, there was a significant difference in infraspinous muscle, supraspinous muscle and serratus anterior muscle but not in the middle deltoid muscle $(p<.05)(p<.001)$ Table 2.

Table 2. Comparison of changes in median frequency value before and after repair in Experimental group

\begin{tabular}{|c|c|c|c|c|c|}
\hline \multirow{3}{*}{ items } & \multicolumn{2}{|c|}{$\begin{array}{l}\text { Experimental } \\
\text { group }(\mathrm{n}=32)\end{array}$} & \multirow{3}{*}{$\begin{array}{l}\text { Shapiro- } \\
\text { Wilk }\end{array}$} & \multirow{3}{*}{$\mathrm{T}$} & \multirow{3}{*}{$\mathrm{P}$} \\
\hline & Pre-test & Post-test & & & \\
\hline & $\mathrm{M} \pm \mathrm{SD}$ & $\mathrm{M} \pm \mathrm{SD}$ & & & \\
\hline Infraspinatus & $\begin{array}{l}99.93 \\
\pm 8.34\end{array}$ & $\begin{array}{l}90.43 \\
\pm 4.14\end{array}$ & .616 & 5.652 & $.000^{*}$ \\
\hline Supraspinatus & $\begin{array}{l}100.84 \\
\pm 6.94\end{array}$ & $\begin{array}{l}92.98 \\
\pm 4.38\end{array}$ & .976 & 5.278 & $.000^{*}$ \\
\hline $\begin{array}{l}\text { Serratus } \\
\text { anterior }\end{array}$ & $\begin{array}{l}96.22 \\
\pm 9.84\end{array}$ & $\begin{array}{l}91.54 \\
\pm 11.8\end{array}$ & .585 & 2.466 & $.021^{*}$ \\
\hline $\begin{array}{l}\text { Middle } \\
\text { deltoid }\end{array}$ & $\begin{array}{l}93.62 \\
\pm 8.89\end{array}$ & $\begin{array}{l}96.84 \\
\pm 8.24\end{array}$ & .122 & -1.776 & .087 \\
\hline
\end{tabular}

Comparison of changes in median frequency value in shoulder-surrounding muscles before repair for Experimental group

This study compared the median frequency values in muscles before repair. As a result, there was a significant difference in comparing median frequency values in infraspinous muscle, supraspinous muscle, serratus anterior muscle, and middle deltoid muscle $(p<.01)$ Table 3.

As a result of post hoc on the changes in median frequency in muscles before repair, such a change in the middle deltoid muscle appeared smaller than that of the supraspinous muscle Table 3.

Table 3. Comparison of changes in median frequency value in shoulder-surrounding muscles before repair for Experimental group

\begin{tabular}{|c|c|c|c|c|}
\hline Items & $\begin{array}{c}\text { Experimental } \\
\text { group }(\mathrm{n}=32)\end{array}$ & $\mathrm{F}$ & $\mathrm{P}$ & Post-hoc \\
\hline
\end{tabular}

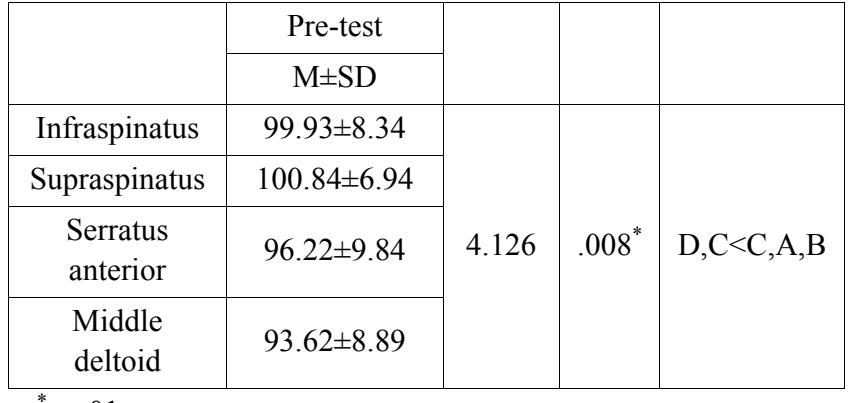

${ }^{*} \mathrm{p}<.01$

A: Infraspinatus muscle, B: Supraspinatus muscle,

C: Serratus anterior muscle, D: Middle deltoid muscle

Comparison of median frequency values in shouldersurrounding muscles after repair for Experimental group

After repair, median frequency values in muscles were compared. As a result, there was a significant difference in comparing median frequency values in infraspinous muscle, supraspinous muscle, serratus anterior muscle and middle deltoid muscle $(p<.05)$ Table 4.

As a result of post hoc on the changes in median frequency in muscles before repair, such a change in the infraspinous muscle appeared smaller than that of the middle deltoid muscle Table 4.

Table 4. Comparison of median frequency values in shouldersurrounding muscles after repair for Experimental group

\begin{tabular}{|c|c|c|c|c|}
\hline \multirow{3}{*}{ Items } & $\begin{array}{l}\text { Experimental } \\
\text { group }(n=32)\end{array}$ & \multirow{3}{*}{$\mathrm{F}$} & \multirow{3}{*}{$P$} & \multirow{3}{*}{ Post-hoc } \\
\hline & Pre-test & & & \\
\hline & $\mathrm{M} \pm \mathrm{SD}$ & & & \\
\hline Infraspinatus & $90.43 \pm 4.14$ & \multirow{4}{*}{3.461} & \multirow{4}{*}{$.019^{*}$} & \multirow{4}{*}{$\begin{array}{c}\mathrm{A}, \mathrm{C}, \mathrm{B}< \\
\mathrm{C}, \mathrm{B}, \mathrm{D}\end{array}$} \\
\hline Supraspinatus & $92.98 \pm 4.38$ & & & \\
\hline $\begin{array}{l}\text { Serratus } \\
\text { anterior }\end{array}$ & $91.54 \pm 11.8$ & & & \\
\hline $\begin{array}{l}\text { Middle } \\
\text { deltoid }\end{array}$ & $96.84 \pm 8.24$ & & & \\
\hline
\end{tabular}

A: Infraspinatus muscle, B: Supraspinatus muscle,

C: Serratus anterior muscle, D: Middle deltoid muscle

\section{DISCUSSION}

With the recent development of medical diagnostic technique, it is known that muscular skeletal patients increase due to various sociocultural factors and patients with shoulderrelated disease is increasing rapidly. In particular, rotator cuff tear occurs most frequently. This rotator cuff tear is accompanied by reduction in the range of motions in joints, generally due to pain around shoulder, muscular atrophy, severe weakness and stiffness [27]. So, after repair, patients need to maintain about six-week fixation using ultra-sling for arms, in order to limit active motion [28]. However, it is said that such a fixation causes muscular activity in muscles surrounding the repaired portion and brings about changes in 
myotilty [14]. If muscular activity is reduced, it is closely related to muscle fatigue and this affects median frequency value because EMG spectrum can be shifted if there are changes in the width in measuring EMG [29]. Therefore, this study aimed to measure EMG signals around the muscles surrounding shoulder during fixation by aid before and after repair in patients who received a rotator cuff repair, identify the impact of muscles on muscular fatigue. This also presents a foundation for studying efficient remedial exercises in patients who would receive a repair in the future.

To predict muscular fatigue, EMG frequency spectrum analysis is used to extract various frequency components, and the commonly-used variables are median frequency and average frequency [30]. Various previous researchers reported various results on median frequency and average frequency, but in particular, median frequency is considered to be the scale that reflects the variation in frequency very well in EMG spectrum analysis of muscle fatigue. So this study used median frequency components as the variable for analysis.

In an experiment to identify the correlation between muscular strength and fatigue, it was found that in case of maximum voluntary contraction of people who showed no muscle fatigue, about $95 \%$ force could be given, whereas in case of maximum voluntary contraction of patients who showed fatigue, about $46 \%$ force was given in comparison. This suggests that there is a correlation between muscular strength and fatigue. This also suggests that such a result is important for rehabilitation or clinical treatment because it may affect fatigue during therapeutic exercise, a rehabilitation process after repair [10].

In general, muscle fatigue refers to reduced efficacy and loss of reaction, which also means that the occurrence of muscular strength is decreased or muscular strength cannot be maintained like that in the beginning. As criteria to determine exercise method and strength, local fatigue of muscle is very important, and if muscle fatigue increases, EMG spectrum moves from high frequency to low frequency [31]. Kim JangGyu [32] reported that as a result of comparing the changes in muscular fatigue after repair in initial remedial exercise group compared to the later remedial exercise group, the median frequency value appeared low in the later remedial exercise group, and Nam Gi-Sung et al. [33] reported that in analyzing the fatigue in back muscle against abdominal muscle in chronic back pain patients, the median frequency value was lower in the back pain patients due to disused back muscle and abdominal muscle. In addition, Jung Lee-Jung et al. [34] reported that as a result of comparing muscular fatigue in wrist bending, extending, and contracting in hemiplegic patients compared to healthy people using EMG, the median frequency value appeared lower in hemiplegic patients and thus hemiplegic patients had more muscle fatigue in their wrist. Such findings suggest that the reduction in high frequency components of median frequency value in EMG spectrum proves the presence of muscle fatigue [35]. This study shows that due to fixation after rotator cuff infraspinous muscle repair, infraspinous muscle appeared from $99.9 \mathrm{~Hz}$ to $90.4 \mathrm{~Hz}$, supraspinous muscle from $100.8 \mathrm{~Hz}$ to $93 \mathrm{~Hz}$, and serratus anterior muscle from $96.2 \mathrm{~Hz}$ to $91.5 \mathrm{~Hz}$, which were significantly low. So this study supported the previous research. However, the middle deltoid muscle showed no significant difference from $93.6 \mathrm{~Hz}$ to $96.8 \mathrm{~Hz}$, which suggests that as extending aid is removable for continuous shoulder-joint passive motion, median frequency value is high in middle deltoid muscle than other muscles and this is less affected by muscular fatigue. On the other hand, it is considered that the diameter of muscle fiber is reduced due to non-use of other muscles and resistance against fatigue becomes relatively weakened and thus median frequency value is reduced. In addition, in post hoc of muscles before repair, the median frequency value appeared much lower in middle deltoid muscle than in supraspinous muscle, and in post hoc of muscles after repair, the median frequency value appeared much lower in infraspinous muscle than in middle deltoid muscle. Kupa et al. [36] reported that disuse atrophy of muscle fiber, denaturalization of muscle fiber, and lateral reinnervation after repair caused changes in median frequency value, which supports our study findings.

It is reported that reduction in median frequency in muscles around shoulder after rotator cuff repair indicates reduction in maximum voluntary contraction in maintaining muscular activity [37], and such weakness in mobilizing motor units causes reduction in muscular activity because loss of network of excitement between spinal segment motor neurons and interneurons from cerebral nerves causes reduction in threshold value of force and adjustment [34]. The characteristics of activity in motor units do not change in general, but among motor units, type I fiber appears from the range of low frequency and type II fiber appears from the range of high frequency because it is mobilized more rapidly when generating a higher and stronger force. Therefore, it is considered that due to fixation after repair, low average frequency values in muscles around shoulder appeared in the range of relatively lower frequency because of low excitement rate and discontinuous contraction of mobilized units and middle deltoid muscle appeared in the range of relatively higher frequency rate compared to the other muscles because of removable extending aid and so it seems to be necessary to develop remedial exercise program in the order of supraspinous muscle, serratus anterior muscle, and infraspinous muscle utilizing middle deltoid muscle with the least muscular fatigue in a 3-week rehabilitation process.

Therefore, as shown in previous research indicating that the phenomenon of median frequency value being changed from range of high frequency to that of low frequency is suggestive of muscular fatigue, this study also shows that the median frequency value shifted from high frequency to low frequency (low frequency shifting) as a result of analyzing the changes in median frequency value, using EMG spectrum of muscles around shoulder in patients after fixating the repaired portion by performing continuous shoulder joint passive motion, an exercise method of 3-week general rehabilitation at a physical therapy room after repair and wearing aid. Therefore, such findings obtained from EMG spectrum analysis after repair are considered to be a big help for developing efficient remedial exercise program. 


\section{CONCLUSION}

This study aimed to identify the changes in muscular fatigue through median frequency analysis of muscles surrounding the shoulder using EMG spectrum after rotator cuff repair and provide basic data for developing efficient remedial exercise programs at clinical settings.

As a result, for three weeks after rotator cuff repair, there was a significant difference in median frequency in infraspinous muscle, supraspinous muscle, and serratus anterior muscle in patients who received continuous shoulder-joint passive motion, which showed that they had muscular fatigue, but there was no significant difference in median frequency value in middle deltoid muscle. This shows that due to fixation with aid, muscular atrophy occurred in infraspinous muscle, supraspinous muscle and serratus anterior muscle, which caused muscular fatigue, and middle deltoid muscle was less affected by muscle fatigue than other muscles because extending aid was attached to the muscle for 3-week continuous shoulder-joint passive motion. In addition, as a result of post hoc of muscles after repair, muscular fatigue appeared less in middle deltoid muscle than in supraspinous muscle and also as a result of post hoc of muscles after repair, muscular fatigue appeared less in infraspinous muscle than in middle deltoid muscle, which suggests that these are the result of atrophy and denaturalization of muscular fiber and lateral reinnervation.

Therefore, it is desirable to study the development of efficient remedial exercise program interventions in the order of supraspinous muscle, serratus anterior muscle, and infraspinous muscle by utilizing middle deltoid muscle with the least muscular fatigue in 3-week rehabilitation process in patients who received rotator cuff repair at clinical settings.

\section{ACKNOWLEDGEMENT}

This reaserch was supported by reaserch funds of Sehan university in 2014

\section{REFERENCES}

[1] L. C. Hall, E. E. Middlebrook, and C. R. Dickerson, "Analysis of the influence of rotator cuff impingements on upper limb kinematics in an elderly population during activities of daily living," Clinical biomechanics, vol. 26, no. 6, 2011, pp. 579-584.

[2] M. A. Smith and W. T. Smith, "Rotator cuff tears: an overview," Orthopaedic nursing, vol. 29, no. 5, 2010, pp. 319-322.

[3] J. S. Abrams, "Arthroscopic approach to massive rotator cuff tears," Instructional course lectures, vol. 55, 2006, pp. 59-66.

[4] G. R. Williams, C. A. Rockwood, L. U. Bigliani, J. P. Iannotti, and W. Stanwood, "Rotator cuff tears: why do we repair them?," The Journal of bone and joint surgery American volume, vol. 86, no. 12, 2004, pp. 2764-2776.
[5] S. E. An and B. Kim, "The Effect of Rehabilitation exercise programs on Recoverability of muscle functions following the Arthroscopy surgery performed on Rotator cuff tear," Journal of sport sociology, vol. 11, no. 1, 2013, pp. 301-309.

[6] R. F. Escamilla and J. R. Andrews, "Shoulder muscle recruitment patterns and related biomechanics during upper extremity sports," Sports medicine, vol. 39, no. 7, 2009, pp. 569-590.

[7] P. O. Zingg, B. Jost, A. Sukthankar, M. Buhler, C. W. Pfirrmann, and C. Gerber, "Clinical and structural outcomes of nonoperative management of massive rotator cuff tears," The Journal of bone and joint surgery, vol. 89, no. 9, 2007, pp. 1928-1934.

[8] A. L. Seitz, P. W. McClure, S. Finucane, N. D. Boardman 3rd, and L. A. Michener, "Mechanisms of rotator cuff tendinopathy: intrinsic, extrinsic, or both?," Clinical biomechanics, vol. 26, no. 1, 2011, pp. 1-12.

[9] D. Celik, B. Sirmen, and M. Demirhan, "The relationship of muscle strength and pain in subacromial impingement syndrome," Acta orthopaedica et traumatologica turcica, vol. 45, no. 2, 2011, pp. 79-84.

[10] S. K. Stackhouse, M. R. Stapleton, D. A. Wagner, and P. W. McClure, "Voluntary activation of the infraspinatus muscle in nonfatigued and fatigued states," Journal of shoulder and elbow surgery, vol. 19, no. 2, 2010, pp. 224229.

[11] M. A. Wirth, C. Basamania, and C. A. Rockwood, "Nonoperative management of full-thickness tears of the rotator cuff," The Orthopedic clinics of North America, vol. 28, no. 1, 1997, pp. 59-67.

[12] N. S. Jo, "Physical Examination of the Elbow," Korean Shoulder and Elbow Society Training lecture, 2009, pp. 46-57.

[13] G. M. Gartsman, "All arthroscopic rotator cuff repairs," The Orthopedic clinics of North America, vol. 32, no. 3, 2001, pp. 501-510.

[14] J. Gondin, M. Guette, and N. A. Maffiuletti et al, "Neural activation of the triceps surae is impaired following 2 weeks of immobilization," European journal of applide physiology, vol. 93, no. 3, 2004, pp. 359-365.

[15] B. K. Downie and B. S. Miller, "Treatment of rotator cuff tears in older individuals: a systematic review," Journal of shoulder and elbow surgery, vol. 21, no. 9, 2012, pp. 1255-1261.

[16] L. A. Wojcik, M. A. Nussbaum, D. Lin, P. A Shibata, and M. L. MaDiqan, "Age and gender moderate the effects of localized muscle fatigue on lower extremity joint torques used during quiet stance," Human movement science, vol. 30, no. 3, 2011, pp. 574-583.

[17] T. S. Ellenbecker, Shoulder rehabilitation: Non-operative treatment, New York: Thieme medical publishers, 2005, pp. $72-74$.

[18] J. C. Na, Exercise physiology, seoul : Daekyung, 2004, pp. 118-143.

[19] V. A. Scholtes, J. G. Becher, A. Beelen and G. J Lankhorst, "Clinical assessment of spasticity in children with cerebral palsy: a critical review of available 
instruments," Developmental medicine and child neurology, vol. 48, no. 1, 2006, pp. 64-73.

[20] C. Gerber, D. C. Meyer, A. G. Schneeberger, H. Hoppeler, and B. von Rechenberg, "Effect of tendon release and delayed repair on the structure of the muscles of the rotator cuff: an experimental study in sheep," The Journal of bone and joint Surgery American volume, vol. 86, no. 9, 2004, pp. 1973-1982.

[21] R. B. Salter, D. F. Simmonds, B. W. Malcolm, E. J Rumble, D. MacMicheal, and N. D Clements, "The biological effect of continuous passive motion on the healing of full-thickness defects in articular cartilage. An experimental investigation in the rabbit," The Journal of bone and joint surgery American volume, vol. 62, no. 8, 1980, pp. 1232-1251.

[22] C. A. Murphy, W. J. McDermott, R. K. Petersen, S. E. Johnson, and S. A. Baxter, "Electromyographic analysis of the rotator cuff in postoperative shoulder patients during passive rehabilitation exercises," Journal of shoulder and elbow surgery, vol. 22, no. 1, 2013, pp. 102-107.

[23] A. Gür, M. Karakoç, K. Nas, R. Cevik, J. Sarac, and E. Demir, "Efficacy of low power laser therapy in fibromyalgia: a single-blind, placebo-controlled trial," Lasers in medical science, vol. 17, no. 1, 2002, pp. 57-61.

[24] J. W. Lee, The effect of neural adaptation and change of skeletal muscle architecture by electrical stimulation, master's dissertation, Dongshin University, 2007.

[25] J. H. Kim, H. H. Choi, and J. I. Youn, "Muscle Fatigue Analysis Based on Electromyography Signals for The Evaluation of Low-Level Laser Therapy," Journal of biomedical engineering research, vol. 32, no. 4, 2011, pp. 319-327.

[26] P. J. Sparto, M. Parnianpour, E. A. Barria, and J. M. Jaqadeesh, "Wavelet and short-time Fourier transform analysis of electromyography for detection of back muscle fatigue," IEEE Transactions on rehabilitation engineering, vol. 8, no. 3, 2000, pp. 433-436.

[27] B. R. Neri, K. W. Chan, and Y. W. Kwon, "Management of massive and irreparable rotator cuff tears," Journal of shoulder elbow surgery, vol. 18, no. 5, 2009, pp. 808-818.

[28] S. J. Seok, A study on the concerns of patient with the rotator cuff repair, master's dissertation, Konkuk University, 2011.

[29] M. H. Kang, "The analysis of lumbar extensor fatique during trunk exercise in low back pain patients," The korean journal of physical education, vol. 20, no. 1, 2011, pp. 1085-1093.

[30] R. Casale, M. Buonocore, A. Di Massa, and C. Setacci, "Electromyographic signal frequency analysis in evaluating muscle fatigue of patients with peripheral arterial disease," Archives of physical medicine and rehabilitation, vol. 75, no. 10, 1994, pp. 1118-1121.

[31] H. C. Kim, A study on muscle fatigue and activity and age related changes during static exercise of shoulder muscles using theraband, master's dissertation, Industry University, 2009.

[32] J. K. Kim, Comparison of knee myofuction according to rehabilitation exercise program participation period after anterior cruciate ligament reconstruction, master's dissertation, Kook-min University, 2009.

[33] K. S. Nam, Y. H. Lee, C. H. Yi, and S. H. Cho, "A Study of Muscle Fatigue in Lumbar and Abdominal Muscles in Patients with Chronic Low Back Pain by Electromyographic Power Spectral Analysis," Korean research society of physical Therapy, vol. 6, no. 2, 1999, pp. 16-131.

[34] Y. J. Chung, S. H. Cho, O. Y. Kwon, and Y. H. Lee, "Characteristics of initiation and termination of muscle contraction in early hemiparetic wrists:Analysis of median frequency," Physical Therapy Korea, vol. 13, no. 1, 2006, pp. 38-46.

[35] M. Y. Lee, Electromyographic Study on Lumbar Extensor Activity and Fatigue during Trunk Flexion and Extension Exercise, dactoral dissertation, Kon-Kuk University, 2000.

[36] E. J. Kupa, S. H. Roy, S. C. Kandarian, and C. J. De Luca, "Effects of muscle fiber type and size on EMG median frequency and conduction velocity," Journal of applied physiology(Bethesda, Md:1985), vol. 79, no. 1, 1995, pp. 23-32

[37] N. A. Riley and M. Bilodeau, "Changes in upper limb joint torque patterns and EMG signals with fatigue following a stroke," Disability and rehabilitation, vol. 24, no. 18, 2002, pp. 961-969.

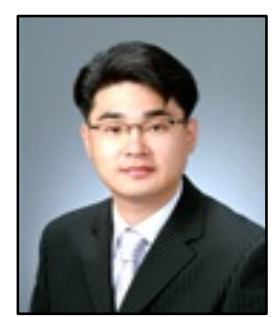

\section{Jeong-Il Knag}

$\mathrm{He}$ received the Ph.D in Hospital of Korean Medicine from Wonkwang University Graduate School, Korea in 2007. Since then, he was completed March 2008 until now, being Sehan University Professor of Physical Therapy. His research interests include Diseasespecific physical therapy, Physical therapy diagnostics and Health statistics.E-Mail: jikang@sehan.ac.kr

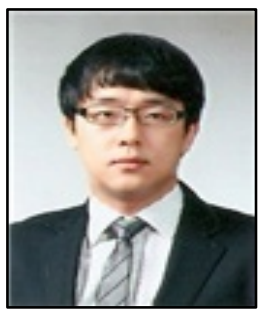

\section{Young-Jun Moon}

He received the B.S. in physical therapy from Sehan University, Korea in 2011. He received the M.S. in physical therapy from Sehan University Graduate School, Korea in 2013. He is currently Doctorate course in physical therapy department of Sehan University. His research interests include Hydrotherapy, Light therapy, Orthotics. E-Mail: tkfkdgo0328@naver.com 